\title{
An optical sensor for studying hemocompatibility of biomaterials in a flow system
}

\author{
Johanna Hutterer ${ }^{1}$, Günter Gauglitz ${ }^{1}$ \\ ${ }^{1}$ Department of Chemistry, Eberhard Karls University, Auf der Morgenstelle 18, 72076 Tübingen, \\ Germany \\ Johanna.hutterer@iptc.uni-tuebingen.de
}

\begin{abstract}
When implants get into contact with body fluids such as blood, lymph or saliva, proteins start to adhere to their surface within milliseconds. The amount, type and possible conformational changes of the adhering proteins initiate various processes, such as platelet accumulation, activation of immunological cascades, formation of foreign body giant cells or apoptosis of adherent cells [1]. These unintended interactions of a foreign material with the human body can inter alia - result in implant failure.

The here presented sensor has been developed to be particularly suitable for testing hemocompatibility. The formation of a protein film that develops on the sensor surface is recorded using reflectometric interference spectroscopy [2]. This label-free and temperature independent technique monitors the change in optical thickness - the product of physical thickness and the reflective index - caused by association or disassociation processes. By using a semi-steady flow of the protein solution across the sensor surface, the fluid dynamic properties of blood vessels are mimicked. The influence on the formation of protein films of different commonly used materials in cardio-vascular implants, such as polyethylene glycol and polyurethane, and furthermore a biomimicking phospholipid membrane surface was evaluated.

In the future, the here presented method can be extended to adherence analysis of further blood components, such as platelets, erythrocytes and lymphocytes. By testing the adherence of these cellular blood components individually or in combination with other blood components, a more complete picture of the body response to cardio-vascular implant surfaces could be achieved.
\end{abstract}

Keywords: optical sensor, biomaterial,biomolecular interaction analysis, blood compatibility,reflectometric interference spectroscopy

\section{Background}

Implanting foreign materials into a human body, which is unavoidable in many cases, is still associated with additional risks compared to reconstructive approaches. For example, for patient with an artificial heart valve implanted, prosthesis associated complications range from thromboembolisms, prosthetic valve infections and tissue overgrowth to mechanical valve failure [1]. If a foreign material is implanted into the body, numerous signaling cascades and processes are initiated resulting in either acceptance or rejection of the implant by the human individual. This process is referred to as foreign body reaction (Fig. 1). When implants get into contact with body fluids such as blood, lymph or saliva, proteins start to adhere to their surface within milliseconds. The amount, type and possible conformational changes of the adhering proteins trigger various processes, such as platelet accumulation, activation of immunological cascades, formation of foreign body giant cells or apoptosis of adherent cells [2]. These unintended interactions of a foreign material with the human body can result in implant failure. Considering these facts, the here presented optical sensor aims to assist in understanding basic mechanisms in implant rejection by investigating the initial adsorption of blood proteins to the implant surface. To study these adsorption processes several methods including surface plasmon resonance, ellipsometry, infrared spectroscopy, atomic force microscopy and optical microscopy are available. However, the application of the here presented optical method offers further insights from a physicalchemical perspective. 


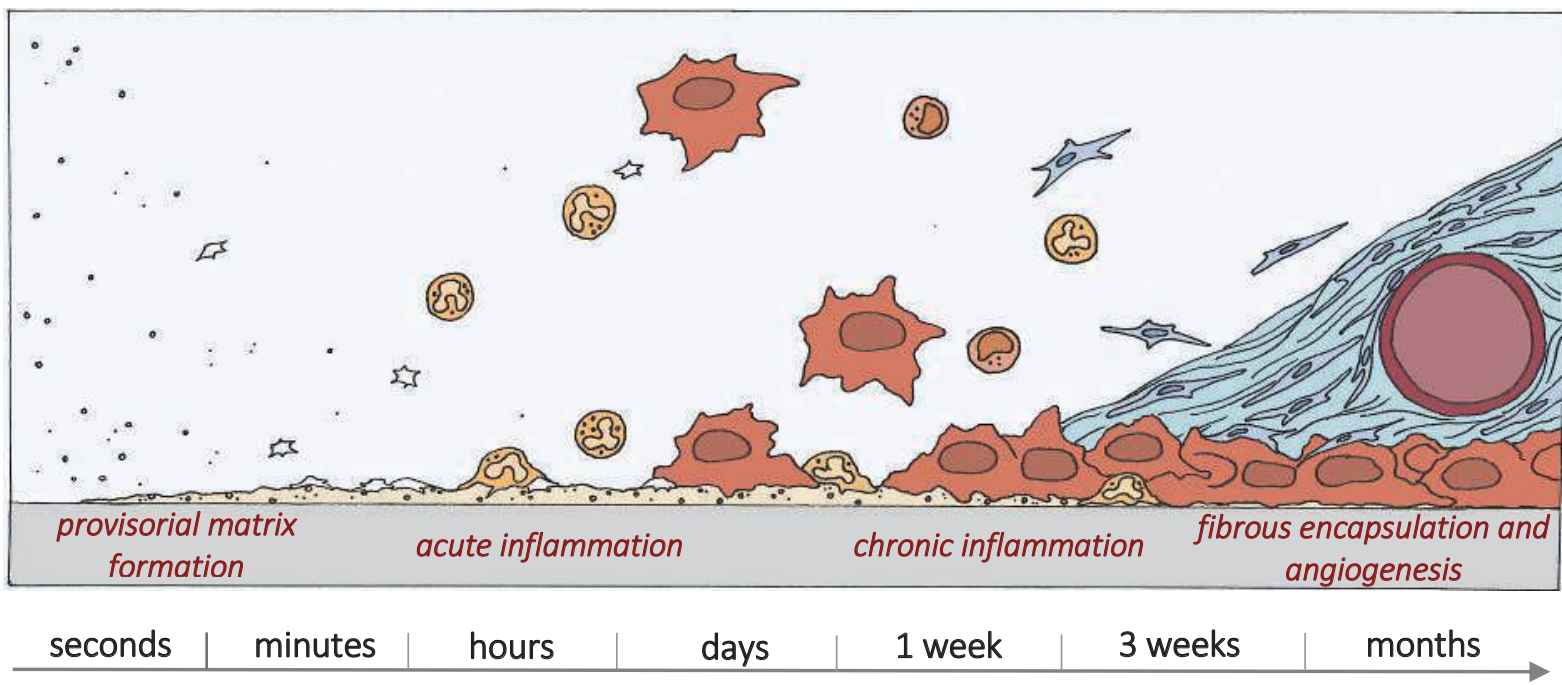

Fig. 1: foreign body reaction. Chronical sequence of interactions taking place at the implant surface after implantation.

\section{Method and Materials}

\section{Reflectometric Interference Spectroscopy}

The formation of a protein film that develops on the sensor surface is recorded using reflectometric interference spectroscopy (RIfS) [3]. This label free and temperature independent technique monitors the change in optical thickness $(\Delta n d)$ - the product of physical thickness and the reflective index caused by association or disassociation.

Using white light, the glass transducer is illuminated from the bottom. At all interphases the light is partly transmitted and partly reflected. Here, multiple reflections are caused by the formation of a protein film on the surface, the interfaces between transducer and surface modifications and by a thin tantalum pentoxide layer in the transducer that is used to enhance the reflectivity of the system. The reflected secondary beams superimpose and form a characteristic interference spectrum that is captured by a spectrometer (Fig 2.1). Binding events at the investigated surface lead to a shift of the interference spectrum (Fig. 2.2). By monitoring the displacement of an extrema of the interference spectrum the $\Delta$ nd can be calculated. Thereby, association and dissociation processes at the surface can be studied in a temporally resolved manner (Fig. 2.3).

\section{Surface Modifications}

The surface of the silicon dioxide transducer was modified to study the interactions of polyethylene glycol (PEG), polyurethane (PU),
1)
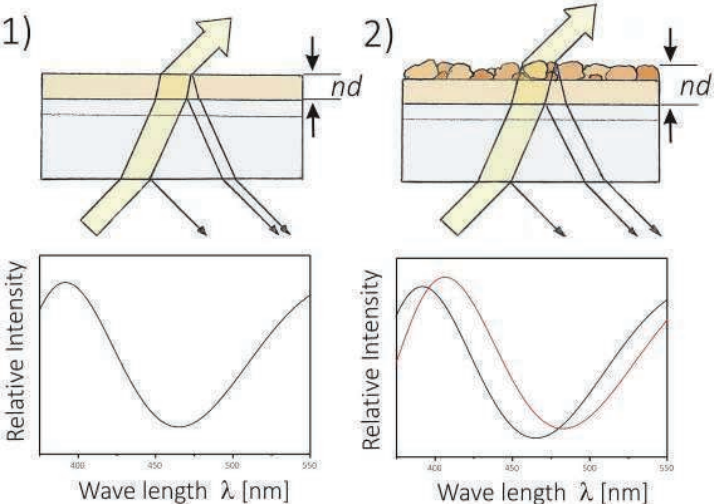

3)

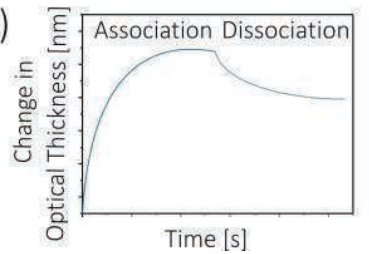

Fig. 2: RIfS detection principle. 1) initial sensor surface and the characteristic interference spectrum caused by multiple reflections of white light at thin films. 2) Displacement of the characteristic interference spectrum due to binding of particles to the transducer surface. 3) Calculated $\Delta$ nd displayed in a sensorgram.

silicon dioxide and a biomimetic phospholipid membrane with $5 \%$ fetal calf serum (FCS) in phosphor buffered saline (PBS) (Fig. 3).

To investigate the interactions on the glass surface, the silicon dioxide transducer was cleaned with potassium hydroxide and $10 \mathrm{~min}$ with acetone in an ultrasonic bath.

The PEG modification of the glass transducer was achieved by activating the cleaned glass 
transducer with piranha solution. After surface silanization with 3-glycidyloxypropyltrimethoxysilane (GOPTS) diamino-PEG was conjugated [4].

To form a phospholipid membrane the cleaned transducer was modified with dicarboxy-PEG (70 \%) and phosphatidylcholine-PEG (30\%) analogous to the PEG surface modification. Phospholipid vesicles were prepared with phosphatidylcholine $(70 \%)$ and cholesterol (30 $\%)$ using an already published method [5]. The phospholipid vesicles were formed in PBS and were pumped across the phosphatidylcholinePEG surface on which the vesicles accumulated. The deposition of the phospholipid vesicles was monitored using RIfS (results not shown here).

The PU surface was generated via spin coating. A solution of $5 \%$ Pellethane 55DE in $\mathrm{N}, \mathrm{N}$-Dimethylacetamide was spin coated on a cleaned glass transducer at $40 \mathrm{rpm}$ for $40 \mathrm{~s}$. The spin coater chuck and the transducers were heated up to $80^{\circ} \mathrm{C}$.

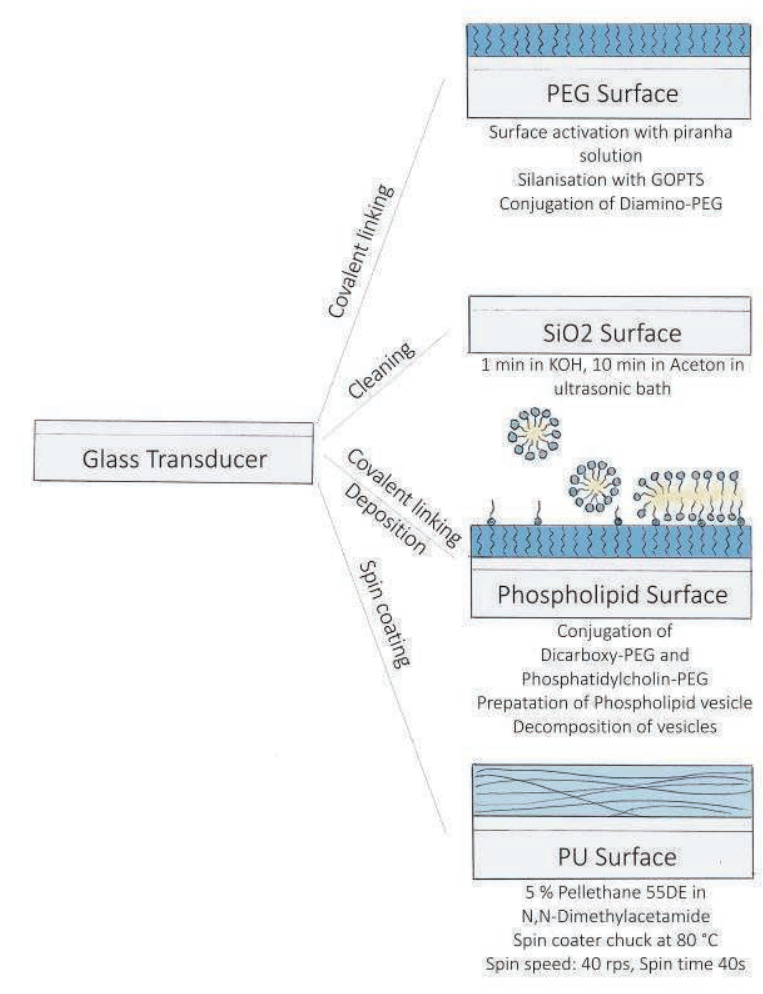

Fig. 3: $\quad$ Surface modifications for interactions studies with blood serum. A silicon dioxide glass transducer surface was modified resulting in a PEG surface, a biomimetic phospholipid membrane surface, a PU surface and a glass surface.

\section{Interaction Studies}

By using a semi-steady flow of the protein solution across the sensor surface, the fluid dynamic properties of blood vessels are mimicked. Fetal calf serum was diluted to $5 \%$ in PBS and pumped across the surface through a flow cell for $700 \mathrm{~s}$. This was followed by applying a constant flow of PBS across the sensor surface to investigate the dissociation process.

After each measurement the surface was restored using a guanidinium hydrochloride solution (6 M, pH 1.5). Triplets were measured on three transducers of each surface modification. As the variations of the triplets were insignificant compared to the variations between the transducers, the here presented means and standard deviations were calculated from the mean of the triplets.

\section{Biomolecular Interaction Analysis (BIA)}

Using the association and dissociation curves recorded via RIfS, kinetical parameters of the interactions at the surface can be calculated [5]. This allows the assessment of the affinity of the investigated proteins to the respective surface.

The binding of the ligand $L$ to the receptor $R$ can be described by a reaction pseudo first order (1) with $R L$ as the ligand-receptor complex:

$$
L+R \leftrightarrow R L
$$

For the simplest case, the concentration of the ligand-receptor complex (2) can be formulated as follows:

$$
\frac{d c_{R L}}{d t}=k_{a} \cdot c_{R} \cdot c_{L}-k_{d} \cdot c_{R L}
$$

with $k_{a}$ and $k_{d}$, the association and dissociation rate, $c_{R}, c_{L}$ and $c_{R L}$ for the concentration of the receptor, the ligand and the receptor-ligand complex, respectively. Thus, the surface loading $\Gamma$ in a heterogenic reaction (3) can be described as:

$$
\frac{d \Gamma(t)}{d t}=k_{a} \cdot c \cdot\left(\Gamma_{\max }-\Gamma(t)\right)-k_{d} \cdot \Gamma(t)
$$

with the maximal surface loading $\Gamma_{\max }$ and the concentration of the ligand in the solution $c$. After integration, the dissociation (4) and the 
association (5) of ligand to the immobilized receptor can be written as:

$$
\begin{aligned}
& \Gamma(t)=\Gamma_{G G} \cdot e^{-k_{d} \cdot t} \\
& \Gamma(t)=\Gamma_{G G} \cdot\left(1-e^{-\left(k_{a} \cdot c+k_{d}\right) \cdot t}\right)
\end{aligned}
$$

Since the used blood serum is composed out of a multitude of different proteins and other components, it is unavoidable to include a second exponential term to parameterize the differences in binding behavior (Fig. 4). The respective formula for association and dissociation are displayed in (6) and (7), respectively. For the dissociation process the variable $B$ was included presenting residual binding to the surface caused by back binding of particles after dissociation.

$$
\begin{aligned}
& \Gamma(t)=\Gamma_{G G 1} \cdot e^{-k_{d 1} \cdot t}+\Gamma_{G G 2} \cdot e^{-k_{d 2} \cdot t}+B \\
& \Gamma(t)=\Gamma_{G G 1} \cdot\left(1-e^{-\left(k_{a 1} \cdot c+k_{d 1}\right) \cdot t}\right) \\
& +\Gamma_{G G 2} \cdot\left(1-e^{-\left(k_{a 2} \cdot c+k_{d 2}\right) \cdot t}\right)
\end{aligned}
$$

As a slight difference in the reflective index of PBS and the FCS solution was observed, first data points of the association process were excluded from the fitting process. By this, the

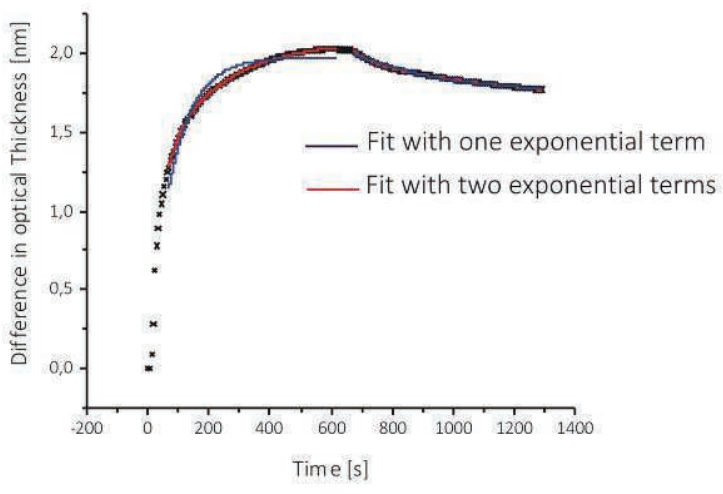

Fig. 4: Fitting of the association and dissociation curve for the calculation of kinetic parameters. Black crosses: exemplary measurement of the association and dissociation of $5 \%$ FCS in PBS on a glass surface. Blue line: Fit of the measurement data with formula (4) and (5) for the association and dissociation process respectively. Red line: Fit resulting from using (6) and (7) as a fitting function for the association and dissociation, respectively. influence of a rapid change of the reflective index - solely caused by the changed solution in the system - on the calculated parameter could be avoided.

The measurements on the polyurethane surface were excluded from BIA calculations as the surface was not stable enough.

\section{Results and Discussion}

The observed $\Delta$ nd for the association of blood proteins was the most prominent on glass surfaces, followed by the biomimetic phospholipid membrane while $\mathrm{PU}$ as well as PEG showed a remarkable small interaction with serum proteins (Fig 4).

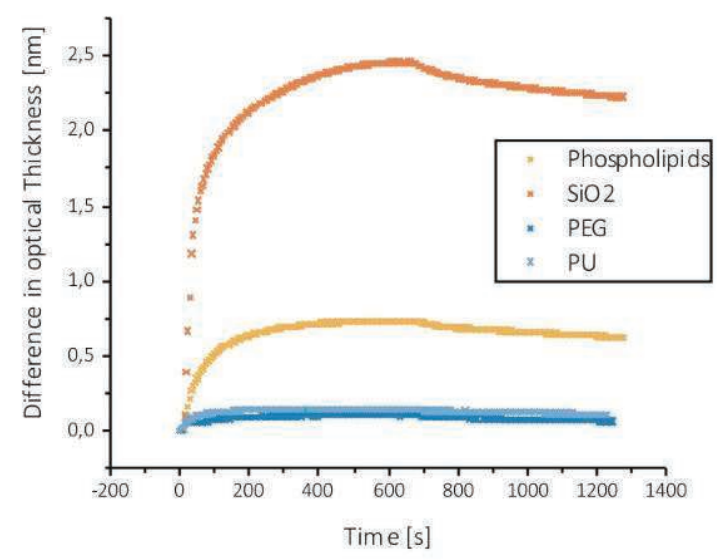

Fig. 5: Sensorgram for association and dissociation of $5 \%$ FCS in PBS on different surfaces. Orange, yellow, bright blue and dark blue crosses depict an exemplary silicon dioxide, phospholipid membrane, PEG and PU surface, respectively.

These findings are supported by the results shown in Fig. 6.1. The mean $\Delta$ nd at the equilibrium surface loading $\left(\Gamma_{G G}\right)$ of the surface was approximately four times higher than on the phospholipid membrane surface and 20 times higher than on the polymers. During the dissociation process the largest $\Delta$ nd was observed for the glass surface between the $\Gamma_{G G}$ and the surface loading after the flow of PBS across the sensor for approximately $600 \mathrm{~s}$. Furthermore, the PEG surface showed a considerably smaller $k_{a 1}$ while showing a larger $k_{d 1}$ compared to the glass surface. 

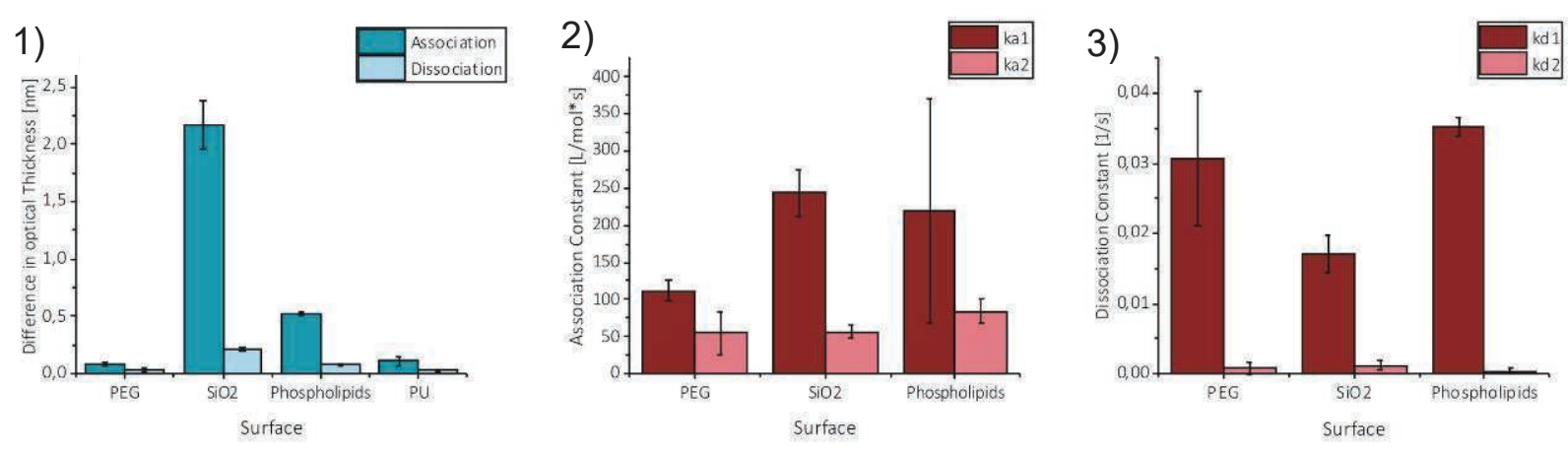

Fig. 6: 1) mean $\Delta$ nd caused by association (dark blue) and dissociation (bright blue) processes by interactions of $5 \%$ FCS in PBS with different surfaces. The $\triangle$ nd representing the association process $\Delta n d_{\text {ass }}$ was calculated by $\Delta n d_{\text {ass }}=\Gamma_{G G}-\Gamma(t=0)$, where the $\Delta$ nd representing the dissociation $\Delta n d_{\text {diss }}$ was calculated by $\Delta n d_{d i s s}=\Gamma_{G G}-\Gamma(t=1300)$. Error bars depict the standard deviation 2) calculated mean ( $n=3) k_{a 1}$ and $k_{a 2}$ using fitting function (7) where error bars depict the standard deviation. 3) calculated mean (n=3) $k_{d 1}$ and $k_{d 2}$ using fitting function (6) where error bars depict the standard deviation.

Also, the phospholipid membrane surface showed a large $k_{d 1}$ compared to the glass surface (Fig. 6.2-3). Thus, these first results indicate that biomaterials showing a good biocompatibility feature a different kinetical behavior compared to materials with rather insufficient bio-compatibility.

\section{Conclusion and Outlook}

The here presented results show that using RIfS for studying adsorption characteristics of serum proteins to biomaterials is reasonable. Differences in the equilibrium surface loadings, measured by $\Delta$ nd, as well as in the kinetical behavior during association and dissociation were shown for different surface modifications.

In the future, the here presented method can be extended to adherence analysis of further blood components, such as platelets, erythrocytes and lymphocytes. By testing the adherence of these cellular blood components individually or in combination with other blood components, a more complete picture of the body response to cardio-vascular implant surfaces could be achieved.

\section{References}

[1] Schoen, Frederick J.; Gotlieb, Avrum, Cardiovascular Pathology (2016); DOI: 10.1016/j.carpath.2016.05.002.

[2] Anderson, James M.; Rodriguez, Analiz; Chang, David T., Innate and Adaptive Immune

Responses in Tissue Engineering (2008);

DOI: 10.1016/j.smim.2007.11.004.
[3] Gauglitz, G.; Brecht, A.; Kraus, G.; Mahm, W., Sensors and Actuators B: Chemical (1993); DOI: 10.1016/0925-4005(93)85234-2.

[4] Mehne, Jochen; Markovic, Goran; Proll, Florian; Schweizer, Nina; Zorn, Stefan; Schreiber,

Frank; Gauglitz, Gunter, Analytical and Bioanalytical Chemistry (2008); DOI: 10.1007/s00216-008-2066-0.

[5] Hilbig, Urs; Bleher, Oliver; Le Blanc, Alexander; Gauglitz, Günter, Analytical and Bioanalytical Chemistry (2012);

DOI: $10.1007 / s 00216-012-5831-z$.

\section{Acknowledgements}

This work was founded by the „Ministerium für Wissenschaft, Forschung und Kunst des Landes Baden-Württemberg" in the context of the doctoral study course "Intelligente Prozessund Materialentwicklung in der Biomateriomics". 